\title{
Site of upper airway obstruction in obstructive apnoea and influence of sleep stage
}

\author{
A.N. Boudewyns*, P.H. Van de Heyning*, W.A. De Backer**
}

Site of upper airway obstruction in obstructive apnoea and influence of sleep stage. A.N. Boudewyns, P.H. Van de Heyning, W.A. De Backer. (CERS Journals Ltd 1997.

ABSTRACT: Various sites along the upper airway (UA) are prone to narrow and collapse in patients affected by obstructive sleep apnoea. Sleep stages may eventually alter these sites. The present study was designed to determine the site(s) of UA obstruction and the influence of sleep stage on the pattern of obstruction.

Twenty eight obstructive sleep apnoea patients underwent UA pressure measurements during polysomnography. Solid-state pressure sensors were located at the nasopharynx, oropharynx, tongue base, hypopharynx and oesophagus and the lower limit of UA obstruction was determined relying on the observed pressure pattern.

The site of UA obstruction varied among consecutive apnoeas in all but two patients. The lower limit of UA obstruction was predominantly located at the nasoand oropharynx. Rapid eye movement (REM) sleep was associated with a tendency for obstruction to extend towards lower levels of the UA and nasopharyngeal occlusion was significantly less observed during REM compared to oropharyngeal obstruction.

Upper airway obstruction involves more than one specific site of the upper airway in the majority of sleep apnoea patients. Obstruction at lower levels of the upper airway is more likely to be observed during rapid eye movement sleep. Eur Respir J 1997; 10: 2566-2572. *Dept of Otorhinolaryngology, Head and
Neck Surgery, and **Dept of Pulmonary Medicine, Antwerp University, Belgium.

Correspondence: W.A. De Backer University Hospital of Antwerp Department of Pulmonary Medicine Wilrijkststraat 10

2650 Edegem

Belgium

Keywords: obstructive sleep apnoea sleep stage

upper airway

Received: November 151996

Accepted after revision July 301997

ANB is an assistant of the Fund for Scientific Research Flanders (FWO), Belgium.
Intermittent closure of the pharyngeal airway resulting in episodes of hypoxaemia and sleep fragmentation, characterize the obstructive sleep apnoea syndrome (OSAS) [1]. OSAS is a common condition, affecting 2-4\% of middle-aged adults and is associated with an increased cardiovascular risk [2, 3]. The cause of upper airway (UA) obstruction is multifactorial and the following factors contribute to the pathogenesis of this condition: anatomic narrowing of the UA; increased collapsibility of UA tissues; reflexes affecting UA calibre; and pharyngeal muscle function.

In an attempt to understand the functioning and behaviour of the UA, various techniques permitting UA characterization have been developed. Among them, nasal endoscopy, cephalometry and UA imaging by computed tomography (CT), are probably the best documented. These techniques are, however, difficult to perform during sleep and it is well known that the UA behaves differently during sleep than during wakefulness. In addition, the results obtained by static techniques such as cephalometry or CT scan do not provide information about UA dynamics. A discrepancy between static (lateral cephalometry, CT scan) and dynamic examinations of the UA (somnofluoroscopy) has been emphasized by PÉPIN et al. [4]. These authors found an extension of airway collapse to the hypopharynx during somnofluoroscopy in 10 of 11 patients whereas CT scan did not show a reduction of this area in six of these patients. A disagreement, with respect to the findings in the hypopharynx between cephalometry (upright position) and somnofluoroscopy was found in seven patients. From these results it becomes evident that dynamic studies of the UA during sleep are necessary to investigate the pattern of UA obstruction and to document the involvement of the hypopharynx. UA pressure measurements provide information about UA dynamics in terms of changes in UA pressure before, during and after the appearance of respiratory events. Results from UA pressure measurements have been reported by several authors using different techniques varying from a fluid-filled catheter [5] to movable catheters [6, 7], a bias flow catheter [8-10] and micropressure sensors [11-15]. Most studies were performed in a limited number of patients $(\mathrm{n}<20)$ except those of KATSANTONIS et al. [15], Scatvedt [13] and Metes et al. [7]. The results of these studies, however, are controversial. ScAtvedT [13] and Tvinnereim and Miljeteig [12] demonstrated that apnoeas may result from obstruction at variable sites in a given patient. In contrast, the other authors concluded that UA obstruction is located at one particular site in the UA which remains the same during consecutive apnoeas.

UA collapsibility is known to vary among different sleep stages [16]. To our knowledge, the influence of sleep stage on the occurrence of obstruction at a particular site of the UA, has not been thoroughly investigated. In the study by KATSANTONIS et al. [15], it is mentioned that the site of collapse remained constant 
throughout sleep stages and positions, but detailed data on this issue were not presented. SHEPARD and THAWLEY [5] studied nine patients during both rapid eye movement (REM) and nonREM (NREM) sleep and observed an extension of the collapse towards more caudal segments of the UA in seven patients. The controversies and questions elicited by these studies prompted us to investigate the site(s) of UA obstruction and the influence of sleep stage in a sufficiently large number of OSAS patients.

\section{Materials and methods}

\section{Study subjects}

The study included 28 male patients, who were middle-aged (50.6 yrs (SD 9.6)) and obese with a body mass index of $31.7 \mathrm{~kg} \cdot \mathrm{m}^{-2}$ (SD 14.2). A diagnosis of OSAS was established for all of them with apnoea+hypopnoea index (AHI) 41.2 (SD 27.9) and mean oxygen saturation during sleep $91.7 \%$ (SD 1.8).

\section{Study design}

UA pressure measurements were performed during sleep in order to determine the site of UA obstruction during obstructive and mixed apnoeas. The involvement of different UA segments and the relationship between sleep stage and site of obstruction was investigated.

\section{Methods}

UA pressure measurements were performed during complete polysomnography (Alice 3 system; Healthdyne technologies, Marietta, GA, USA), with continuous recording of the following parameters: electroencephalography (EEG) (C4/A1 and C3/A2); electro-oculography (EOG); electromyography (EMG) of chin muscles; cardiac frequency; and tibialis anterior muscle EMG. Respiratory effort was measured by thoracoabdominal strain gauges and oxygen saturation by a fingerprobe corrected to a pulse oximeter (Palco laboratories, Santa Cruz, CA, USA). Airflow was measured by a thermistor in 11 patients and by means of a pneumotachograph (model 279331; Hamilton Medical Inc., Reno, NW, USA) in 17 patients. Pressures along the UA were measured by a Camtip pressure sensor microtip catheter (Camtech, Sandvika, Norway). This catheter consists of a thin silicon tube ( $2 \mathrm{~mm}$ diameter) with five solid-state pressure sensors. Four sensors are located in the UA and one sensor is positioned in the midportion of the oesophagus. The UA sensors are located in the following way (fig. 1): one sensor at the posterior choanae (sensor $\mathrm{N}$ ); a second sensor just below the inferior margin of the soft palate, behind the uvula (sensor P); a third sensor is located at the tongue base ( $\mathrm{O}$ sensor); and a fourth at the tip of the epiglottis (sensor L). The sensors were calibrated in a water column before each recording.

The catheter was inserted through the nose into the UA and oesophagus after local anaesthesia of one nos-

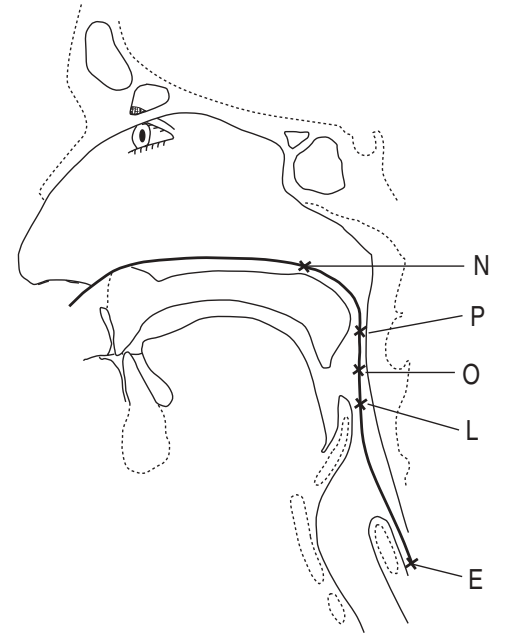

Fig. 1. - Schematic representation of the positions of the different sensors in the upper airway and oesophagus. Sensor N: at the posterior choanae; sensor $\mathrm{P}$ : at the inferior margin of the soft palate behind the uvula; sensor O: at the tongue base; sensor L: at the tip of the epiglottis; sensor E: in the midportion of the oesophagus.

tril with lidocaine $1 \%$ on a cotton swab. The correct placement of the catheter was not verified by imaging techniques. After insertion, the presence of a marker (located at the second sensor) behind the soft palate was verified by visual inspection of the UA and the catheter was repositioned when necessary. Then, the catheter was securely fixed to the skin with tape and correct position of the marker behind the soft palate was checked for a second time.

Sleep tracings were manually scored for sleep stage, respiratory events and site of UA obstruction. Sleep stages were scored as outlined by RECHTSCHAFFEN and KALES [17]. An apnoea was defined as the absence of airflow for at least $10 \mathrm{~s}$. A hypopnoea was scored when a $\geq 50 \%$ reduction of airflow persisted for a minimum of $10 \mathrm{~s}$ and was followed by a $\geq 4 \%$ drop in oxygen saturation and/or arousal. Apnoeas were classified as obstructive, mixed or central according to standard criteria [18]. The AHI was calculated as the number of apnoeas plus hypopnoeas per hour of sleep. The site of UA obstruction was determined for all obstructive and mixed apnoeas. During an apnoea, pressure deflections are absent in the sensors located above the site of obstruction. In those sensors located below the site of obstruction, pressure swings continue to increase during the course of an apnoea. The pattern of pressure deflections in the different sensors during an apnoea allowed us to determine the site of obstruction according to this principle. Extension of an obstruction towards a lower level is inferred when pressure deflections in a particular sensor are present at the onset of apnoea but flatten out or disappear during the course of the event. We used the definitions for the anatomical segments of the UA as proposed by Hudgel [19]. The third sensor separates the oropharynx strictu sensu in an upper and a lower part that we defined as oropharynx and tongue base, respectively. We applied the following terminology to define the site of UA obstruction: between sensor $\mathrm{N}$ and $\mathrm{P}=$ nasopharynx; between sensor $\mathrm{P}$ and $\mathrm{O}=$ oropharynx; between sensor $\mathrm{O}$ and $\mathrm{L}=$ tongue base; between sensor $\mathrm{L}$ and $\mathrm{E}=$ hypopharynx. 


\section{Data analysis}

Four distinct sites of obstruction were identified as specified in the previous section. Considering the number of obstructions at a given site in the UA, the data are presented as the raw data and as data corrected for time spent in a particular sleep stage. Within each patient, there was a difference in the amount of time spent in a particular sleep stage. In order to take this into account, we calculated for each patient the percentage of the total amount of obstructive and mixed apnoeas that occurred during wakefulness, NREM and REM sleep at four sites of the UA under investigation. This permitted the study of the influence of the sleep stage on the site of UA obstruction. The total number of obstructive or mixed apnoeas occurring at different sites of the UA was compared by Friedman analysis of variance (ANOVA). In order to investigate whether sleep stage alone is a predisposing factor for obstruction to occur at a specific site of the UA, the percentage of apnoeas occurring at the nasopharynx during wakefulness, NREM and REM sleep was compared with the percentage of apnoeas occurring at the oropharynx during the same sleep stage (Wilcoxon matched pairs test). Since only a small number of apnoeas was observed to be related to tongue base or hypopharyngeal obstruction, these sites were not considered to study the effect of sleep stage on the site of obstruction. Statistical analysis was performed with the Stat Soft software package (1996, version 5; StatSoft Inc., Tulsa, OK, USA). Statistical significance was assumed at $\mathrm{p}<0.05$.

\section{Results}

Data regarding sleep stage distribution and sleep characteristics are presented in table 1 . The patients spent
Table 1. - Sleep stage distribution and sleep characteristics $(\mathrm{n}=28)$

\begin{tabular}{lr}
\hline Sleep stage & \multicolumn{1}{c}{ Duration } \\
\hline Wakefulness min & $223.5(73.4)$ \\
Stage I NREM min & $69.7(26.7)$ \\
Stage II NREM min & $174.5(59.1)$ \\
Stage III-IV NREM min & $16.2(21.1)$ \\
REM min & $48.8(30.4)$ \\
Total sleep time min & $307.0(72.6)$ \\
Sleep efficiency (total sleep time/time in bed) & $57.4(12.8)$ \\
\hline
\end{tabular}

Values are mean (SD). REM: rapid eye movement; NREM: nonREM.

Table 2. - Combination of the different sites of upper airway (UA) obstruction

\begin{tabular}{|c|c|c|c|c|}
\hline \multirow{2}{*}{$\begin{array}{c}\mathrm{Pt} \\
\mathrm{n}\end{array}$} & \multicolumn{4}{|c|}{ Site of UA obstruction } \\
\hline & Nasopharynx & Oropharynx & $\begin{array}{c}\text { Tongue } \\
\text { base }\end{array}$ & Hypopharynx \\
\hline 1 & $\mathrm{X}$ & & & \\
\hline 1 & & $x$ & & \\
\hline 15 & $\mathrm{X}$ & $\mathrm{x}$ & & \\
\hline 2 & & $\mathrm{x}$ & $\mathrm{X}$ & \\
\hline 7 & $\mathrm{X}$ & $\mathrm{x}$ & $\mathrm{x}$ & \\
\hline 2 & $\mathrm{x}$ & $\mathrm{x}$ & $\mathrm{x}$ & $\mathrm{X}$ \\
\hline
\end{tabular}

Pt: patients; $x$ indicates the site(s) of UA obstruction in each patient.

most time in wakefulness (which also included drowsiness) and stage I-II NREM sleep with little slow-wave and REM sleep.

UA obstruction was located at more than one site in the UA in all but two patients (table 2). In two patients, the primary site of obstruction for consecutive apnoeas was scattered along the entire UA. In some patients, UA obstruction extended towards a lower level of the UA during the same event (fig. 2). The total number of

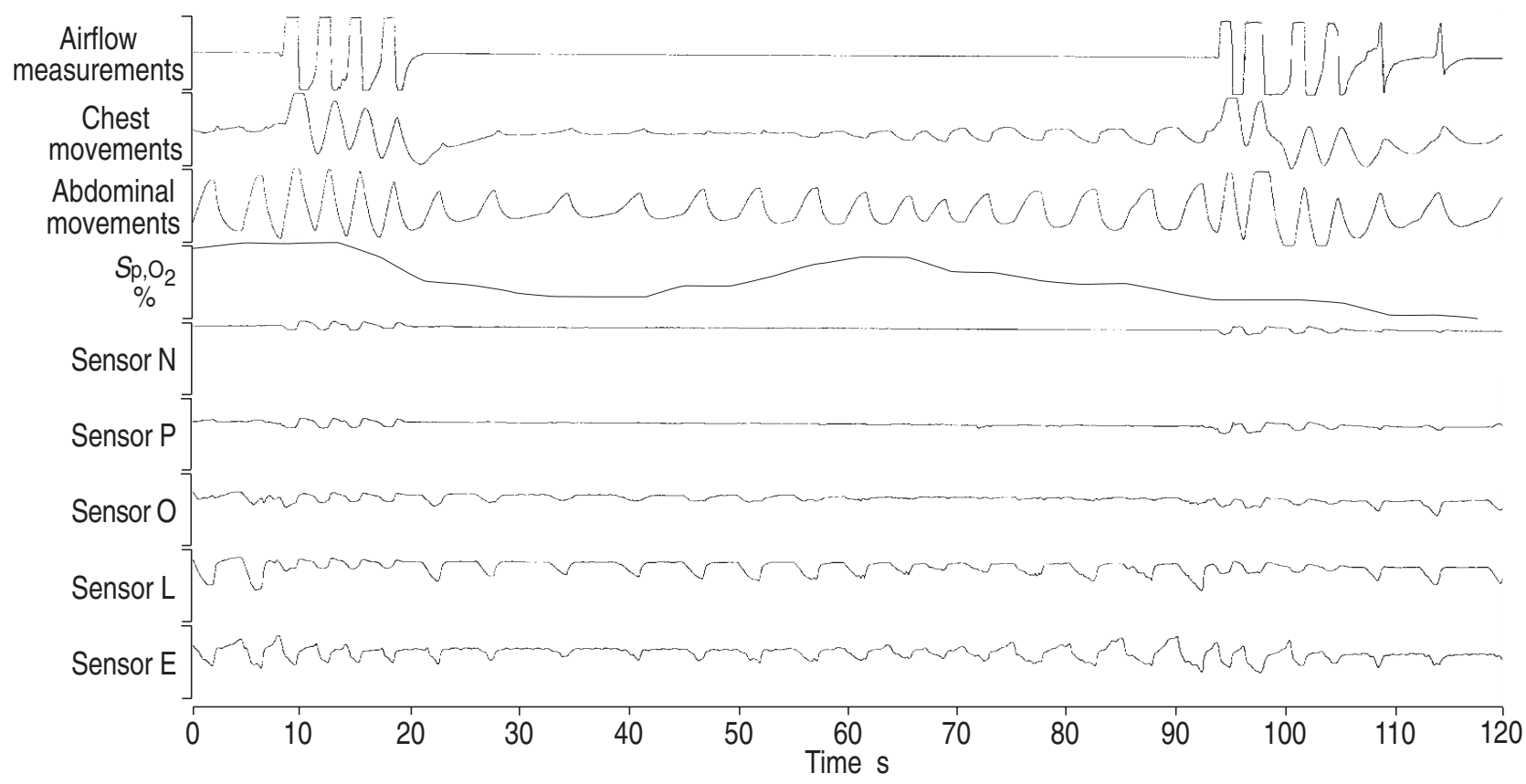

Fig. 2. - Extension of upper airway (UA) obstruction towards a lower site of the UA during the same apnoeic event. In the first half of the apnoea, obstruction is located between the second (sensor P) and third (sensor O) sensor (oropharynx). During the second half, pressure deflections in the third sensor disappear and the obstruction is located between the third and fourth (sensor L) sensor (tongue base). For locations of sensors, see figure $1 . \mathrm{S}_{\mathrm{p}, \mathrm{O}_{2}}$ : pulse oxygen saturation. 


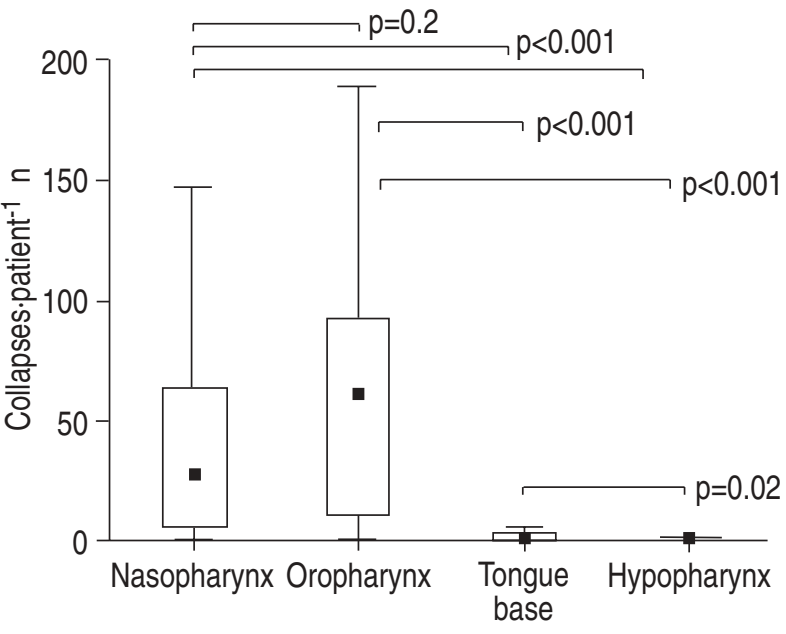

Fig. 3. - Total number of obstructions at a given site of the upper airway for each patient. Shaded boxes represent the median, unshaded boxes represent the interquartile range, and bars represent the nonoutlier maximum and minimum values.

obstructions at a given site of the UA for each patient is displayed in figure 3. Friedman ANOVA ((ANOVA Chi squared $n=28$, degrees of freedom $=3$ ) 51.2, $\mathrm{p}<0.001$ ) followed by Wilcoxon matched pairs test demonstrated that UA obstruction was most likely to occur at the naso- and oropharynx (fig. 3). When the whole study population was considered, the number of obstructions occurring at either the naso- or oropharynx was not sig- nificantly different $(\mathrm{p}=0.2)$. Table 3 displays for each individual patient the percentage of apnoeas occurring at different levels of the UA in relation to sleep stage. The percentage of events occurring at the nasopharynx during wakefulness (including drowsiness) (mean \pm SD $12.3 \pm 18.3)$ or NREM sleep $(26.4 \pm 23.6)$ was not significantly different from the percentage occurring at the oropharynx during wakefulness $(11.9 \pm 10.0 ; \mathrm{p}=0.6)$ or NREM sleep $(34.4 \pm 22.1 ; \mathrm{p}=0.3)$. In contrast, during REM sleep, a higher percentage of apnoeas occurred at the oropharynx $(7.0 \pm 11.7)$ compared to the nasopharynx $(2.8 \pm 1.5 ; \mathrm{p}=0.03)$. In three patients (Nos. 10, 13, and 18; table 3 ), tongue base and/or hypopharyngeal obstruction occurred only during REM sleep and not during NREM sleep or wakefulness.

\section{Discussion}

A first major finding of our investigations is that the lower limit of UA obstruction varied among different apnoeas in all but two patients. This is in agreement with the results of SCATVEDT [13] and TVINNEREIM and Miljeteig [12] who found multiple sites of UA obstruction in seven out of 20 and one out of seven patients, respectively. Our results, however, contrast with those of many others who found the site of obstruction to be fixed during repeated measurements in a given subject.

Table 3. - Percentage of the total amount of obstructive and mixed apnoeas occurring at the nasopharynx, oropharynx, tongue base or hypopharynx for each individual patient in relation to sleep stage

$\%$ total obstructive and mixed apnoeas

\begin{tabular}{|c|c|c|c|c|c|c|c|c|c|c|c|c|c|}
\hline \multirow{2}{*}{$\begin{array}{c}\text { Patient } \\
\mathrm{n}\end{array}$} & \multirow{2}{*}{$\begin{array}{c}\text { Total } \\
\text { apnoeas }\end{array}$} & \multicolumn{4}{|c|}{ Wake } & \multicolumn{4}{|c|}{ NREM } & \multicolumn{4}{|c|}{ REM } \\
\hline & & NP & $\mathrm{OP}$ & TB & HP & NP & OP & TB & HP & NP & $\mathrm{OP}$ & TB & HP \\
\hline 1 & 170 & 3.5 & 7.1 & 0 & 0 & 44.1 & 27.0 & 0 & 0 & 4.7 & 13.5 & 0 & 0 \\
\hline 2 & 124 & 8.1 & 0 & 0 & 0 & 30.6 & 50.0 & 0 & 0 & 0 & 0 & 0 & 0 \\
\hline 3 & 248 & 15.3 & 16.5 & 0 & 0 & 40.3 & 26.2 & 0 & 0 & 1.6 & 0 & 0 & 0 \\
\hline 4 & 6 & 50.0 & 16.7 & 0 & 0 & 33.3 & 0 & 0 & 0 & 0 & 0 & 0 & 0 \\
\hline 5 & 62 & 0 & 27.4 & 0 & 4.8 & 0 & 21.0 & 0 & 0 & 3.2 & 25.8 & 4.8 & 12.9 \\
\hline 6 & 141 & 0.7 & 8.5 & 2.1 & 0 & 1.4 & 70.2 & 12.8 & 0 & 0 & 2.8 & 1.4 & 0 \\
\hline 7 & 127 & 4.7 & 0 & 0 & 0 & 77.9 & 3.1 & 0.8 & 0 & 6.3 & 1.6 & 5.5 & 0 \\
\hline 8 & 94 & 4.2 & 17.0 & 0 & 0 & 2.1 & 73.4 & 0 & 0 & 0 & 3.2 & 0 & 0 \\
\hline 9 & 161 & 18.6 & 16.8 & 0 & 0 & 29.2 & 34.2 & 0 & 0 & 1.2 & 0 & 0 & 0 \\
\hline 10 & 228 & 6.1 & 5.3 & 0 & 0 & 55.3 & 22.8 & 0 & 0 & 3.1 & 6.6 & 0.4 & 0.4 \\
\hline 11 & 93 & 11.8 & 6.5 & 1.1 & 0 & 55.9 & 24.7 & 0 & 0 & 0 & 0 & 0 & 0 \\
\hline 12 & 4 & 25.0 & 0 & 0 & 0 & 50.0 & 25.0 & 0 & 0 & 0 & 0 & 0 & 0 \\
\hline 13 & 91 & 0 & 0 & 0 & 0 & 0 & 56.0 & 7.7 & 0 & 0 & 30.8 & 5.5 & 0 \\
\hline 14 & 42 & 11.9 & 30.9 & 0 & 0 & 11.9 & 45.2 & 0 & 0 & 0 & 0 & 0 & 0 \\
\hline 15 & 205 & 0.1 & 27.8 & 0 & 0 & 6.8 & 58.0 & 0.5 & 0 & 0.5 & 0.5 & 0 & 0 \\
\hline 16 & 57 & 3.5 & 0 & 0 & 0 & 19.3 & 19.3 & 0 & 0 & 47.4 & 10.5 & 0 & 0 \\
\hline 17 & 9 & 0 & 11.1 & 0 & 0 & 0 & 33.3 & 55.6 & 0 & 0 & 0 & 0 & 0 \\
\hline 18 & 136 & 2.9 & 2.2 & 0 & 0 & 16.9 & 57.4 & 0 & 0 & 0 & 19.1 & 1.5 & 0 \\
\hline 19 & 208 & 2.9 & 14.4 & 0 & 0 & 6.3 & 72.1 & 0 & 0 & 0 & 4.3 & 0 & 0 \\
\hline 20 & 27 & 33.3 & 22.2 & 0 & 0 & 18.5 & 25.9 & 0 & 0 & 0 & 0 & 0 & 0 \\
\hline 21 & 491 & 3.3 & 21.6 & 0.4 & 0 & 16.7 & 51.1 & 0.4 & 0 & 0.8 & 3.1 & 2.7 & 0 \\
\hline 22 & 5 & 80.0 & 0 & 0 & 0 & 20 & 0 & 0 & 0 & 0 & 0 & 0 & 0 \\
\hline 23 & 421 & 0.9 & 8.5 & 1.2 & 0 & 6.2 & 66.0 & 5.9 & 0 & 0 & 11.2 & 0 & 0 \\
\hline 24 & 47 & 6.4 & 2.1 & 0 & 0 & 72.3 & 2.1 & 0 & 0 & 8.5 & 8.5 & 0 & 0 \\
\hline 25 & 57 & 36.8 & 21.0 & 0 & 0 & 19.3 & 22.8 & 0 & 0 & 0 & 0 & 0 & 0 \\
\hline 26 & 10 & 0 & 30.0 & 0 & 0 & 0 & 20.0 & 0 & 0 & 0 & 50.0 & 0 & 0 \\
\hline 27 & 199 & 12.6 & 8.5 & 0 & 0 & 39.7 & 33.7 & 0 & 0 & 1.5 & 4.0 & 0 & 0 \\
\hline 28 & 53 & 1.9 & 11.3 & 0 & 0 & 66.0 & 22.7 & 0 & 0 & 0 & 0 & 0 & 0 \\
\hline
\end{tabular}

NP: nasopharynx; OP: oropharynx; TB: tongue base; HP: hypopharynx; REM: rapid eye movement; NREM: nonREM. 
This discrepancy might result from a different interpretation of the data. Two principles can be used in order to determine the site of obstruction. The first is based on the changes in pressure pattern. This technique was proposed by HudgEL [9] and has been applied by others as well as in our present study. The second method is based on the gradient in inspiratory pressures between different sensors. This technique is used by SCATVEDT [13]. Since we interpreted the data in the same way as those authors who found only one site of obstruction, we do not believe that different results reflect discrepancies in interpretation. With respect to data interpretation, another remark needs to be made. When reviewing the literature on UA pressure measurements, it becomes clear that different names are used for the same or overlapping parts of the UA. In the study by SHEPARD and THAWLEY [5], oropharyngeal collapse is said to occur when pressure deflections are absent in two sensors: one located in the nasopharynx at the level of the soft palate and the other located just distal to the inferior margin of the soft palate, at the base of tongue. By contrast, in the study by KATSANTONIS et al. [15], collapse of the oropharyngeal segment is defined as absence of pressure deflections in a sensor located at the posterior choanae. What is defined as nasopharyngeal collapse in our present study corresponds to the oropharyngeal collapse in the study of KATSANTONIS et al. [15], and our definition of oropharyngeal collapse agrees with the definition of oropharyngeal collapse used by SHEPARD and Thawley [5]. The use of different definitions for the UA emphasizes the need for a consensus at this point.

With the exception of the studies by Woodson and Wooten et al. [14] and ScATVEDT [13], previous studies used pressure sensors at only three fixed locations in the UA. The sensors were mostly located in the posterior choanae, at the rim of the soft palate and at the level of the hyoid bone or supraglottis. This allows only classification into retropalatal and retrolingual (hypopharyngeal) obstructors. Obstruction located between the soft palate and tongue base cannot be differentiated from occlusion occurring between the tongue base and the supralaryngeal segment with this technique. We present data obtained with four UA sensors. Our technique might permit more detailed evaluation of the UA and allow to detect additional obstruction sites. We assume that this might explain our observation of multiple obstruction sites.

UA pressure measurements allow for a dynamic study of the UA during sleep and provide information about different UA levels simultaneously. However, the use of pressure sensors to determine the site of obstruction may have some limitations. First, the technique has been criticized since it only permits identification of the lower limit of obstruction [14]. Although manometry allows the measurement of pressure changes at different UA levels simultaneously, it does not identify narrowing at nonobstructive segments of the UA during a respiratory event with complete cessation of airflow. In some patients, small inspiratory pressure fluctuations can be observed upstream from the site of obstruction. This may reflect a subtotal obstruction or reflex contraction of pharyngeal dilator muscles. Combining videoendoscopy with manometry, WoOdson and WoOTEN et al. [14] observed marked narrowing of pharyngeal segments that were not identified as the initial site of obstruction by manometry. CHABAN et al. [6] have tried to overcome this problem by using a movable catheter technique and to determine both the lower and upper limit of obstruction. However, in their study of 10 patients, the upper limit could be located in only two cases due to variability in interaction between the Millar transducer and the pharyngeal wall. Secondly, it is not known to what extent the placement of a catheter in the UA interferes with UA dynamics. Mechanical stimulation of UA receptors or the use of UA anaesthesia (before introduction of the catheter) may change UA characteristics [20,21]. This hypothesis was supported by PÉPIN et al. [22] who summarized the results with respect to the initial site of UA obstruction obtained by videoendoscopy, CT scan or UA pressure measurements. The results from videoendoscopy or CT scan indicated that the initial site of UA obstruction was located in the oropharynx in the majority of patients studied. By contrast, using UA pressure measurements indicated that the obstruction was initiated at the hypopharyngeal level in at least half of the cases. Further studies are necessary to elucidate this controversy. The use of a catheter in the UA may also disturb sleep and result in a decreased sleep efficiency. The observation of a low total sleep time in our study may raise questions about the tolerance of technique by the patient. Three out of 28 patients had a total sleep time of $<200$ min (149, 142 and $153 \mathrm{~min}$, respectively). Previous reports demonstrated that the placement of a catheter in the UA and oesophagus does not induce important additional sleep disturbance in patients with sleep-related breathing disorders [23, 24]. Despite its limitations, the technique of UA pressure measurements may be considered useful to identify the primary site of UA occlusion, to obtain information on multiple airway segments simultaneously during different sleep stages without exposure to radiation.

The knowledge that, in a majority of OSAS patients, UA obstruction occurs at multiple levels, may have important implications for the management of patients with OSAS. SHER et al. [25] reviewed the literature between 1993 and 1996 for papers containing information about the preoperative localization of narrowing or collapse in OSAS patients undergoing uvulopalatopharyngoplasty. Data were available for 168 patients in whom the site of UA obstruction was preoperatively determined by different techniques such as fibreoptic endoscopy (awake or asleep), lateral cephalometric radiography, airway manometry and pharyngeal CT scan. The authors found that the percentage of patients attaining a $50 \%$ decrease in respiratory disturbance index (RDI) and a postoperative RDI of $<20$ (or a $50 \%$ decrease in apnoea index and a postoperative apnoea index $<10$ ) was much higher $(52.3 \%)$ in patients with retropalatal narrowing or collapse than patients with retrolingual or retrolingual and retropalatal narrowing or collapse $(5.3 \%$ $\mathrm{p}<0.0001)$. From these results, the authors concluded that the site of pharyngeal collapse has a marked effect on the outcome of patients undergoing uvulopalatopharyngoplasty.

Our technique of UA pressure measurements, allows for classification of patients into different groups according to the complexity and multiplicity of UA obstruction 
sites during sleep. For each group, the most appropriate treatment needs to be determined. A combination of different surgical procedures or a treatment which can open the UA as a whole, such as continuous positive airway pressure, might be necessary for some patients in order to relieve all sites of obstruction. The influence of new and currently available treatment modalities on these different patterns of UA obstruction, should be thoroughly investigated.

A second major finding of our study considers the influence of sleep stage on the site of UA obstruction. During REM sleep, obstructive and mixed apnoeas occurred more often at the oropharynx compared to the nasopharynx. We noticed that in some apnoeas, the site of obstruction extended towards a lower level in the UA during the same event, and this was more likely to occur during REM sleep. A similar finding was reported by WoODSON and WoOTEN [14]. In three out of 12 patients, these authors observed caudal progression of the obstruction from the palatal region during the course of the event. An extension of the obstruction towards a lower level of the UA can be attributed to either a progressive increase in downstream suction pressure, and thus an increase in transpharyngeal pressure, or to an increase in UA collapsibility or a combination of both. The former is most likely to play a role during NREM sleep. During REM sleep, the oesophageal pressure is less negative compared to NREM sleep and the increase in respiratory effort during the course of an apnoea is less [26]. The occurrence of obstruction at lower levels of the UA during REM sleep compared to NREM sleep may therefore not be attributable to a higher transpharyngeal pressure during REM but may reflect the increased collapsibility of the UA during REM sleep compared to NREM sleep [16]. Because only a limited number of obstructive events were found to be associated with tongue base or hypopharyngeal collapse in our study, the influence of sleep state on the occurrence of obstruction at these sites was not statistically investigated. However, on an individual basis, some patients were found to obstruct at more distal levels along the UA only during REM sleep (patients Nos. 10, 13 and 18; table 3). Our results suggest that REM sleep, is associated with a downward extension of the obstructive site and thus that sleep stage, especially REM sleep may be a predisposing factor for obstruction to occur at a given site of the UA. Other factors such as sleep posture might also have an influence on the site of UA obstruction.

In conclusion, upper airway pressure measurements during sleep demonstrated that the upper airway occludes at multiple sites in the vast majority of patients with obstructive sleep apnoea. Occlusion of the nasoand oropharynx was found to be the predominant cause of obstructive and mixed apnoeas in obstructive sleep apnoea patients. An extension of collapse towards lower levels of the upper airway was most likely to occur during rapid eye movement sleep. These results should be taken into account when selecting obstructive sleep apnoea patients for specific treatment strategies.

Acknowledgements: The authors are grateful to $\mathrm{M}$ Wagemans, W. De Cock, M. Willemen and D. Rouckhout for their technical assistance.

\section{References}

1. Hudgel DW. Mechanisms of obstructive sleep apnea Chest 1992; 101: 541-549.

2. Young T, Palta M, Dempsey J, Skatrud J, Weber S, Badr S. The occurrence of sleep-disordered breathing among middle-aged adults. N Engl J Med 1992; 328: $1230-1235$.

3. Strollo PJ, Rogers RM. Obstructive sleep apnea. $N$ Engl J Med 1996; 334: 99-104.

4. Pépin JI, Ferretti G, Veale D, et al. Somnofluoroscopy, computed tomography and cephalometry in the assessment of the airway in obstructive sleep apneoa. Thorax 1992; 47: 150-156.

5. Shepard JW, Thawley SE. Localization of upper airway collapse during sleep in patients with obstructive sleep apnea. Am Rev Respir Dis 1990; 141: 1350-1355.

6. Chaban R, Cole P, Hoffstein V. Site of upper airway obstruction in patients with idiopathic obstructive sleep apnea. Laryngoscope 1988; 98: 641-647.

7. Metes A, Hoffstein V, Mateika S, Cole P, Haight JSJ. Site of airway obstruction in patients with obstructive sleep apnea before and after uvulopalatopharyngoplasty. Laryngoscope 1991; 101: 1102-1108.

8. Wasicko MJ, Erlichman JS, Leiter JS. Control of segmental upper airway resistance in patients with obstructive sleep apnea. J Appl Physiol 1993; 74: 2694-2703.

9. Hudgel DW. Variable site of airway narrowing among obstructive sleep apnea patients. J Appl Physiol 1986; 61: 1403-1409.

10. Hudgel DW, Harasick T, Katz RL, Witt WJ, Abelson TI. Uvulopalatopharyngoplasty in obstructive apnea. Value of preoperative localization of site of upper airway narrowing during sleep. Am Rev Respir Dis 1991; 143: 942-946.

11. Tvinnereim M, Cole P, Haight JSJ, Hoffstein V. Diagnostic airway pressure recordings in sleep apnea syndrome. Acta Otolaryngol (Stockh) 1995; 115: 449-454.

12. Tvinnereim M, Miljeteig H. Pressure recordings. A method for detecting site of upper airway obstruction in obstructive sleep apnea syndrome. Acta Otolaryngol (Stockh) 1992; Suppl. 492: 132-140.

13. Skatvedt O. Continuous pressure measurements during sleep to localize obstructions in the upper airways in heavy snorers and patients with obstructive sleep apnea syndrome. Eur Arch Otorhinolaryngol 1995; 252: $11-14$.

14. Woodson BT, Wooten MR. A multisensor solid-state pressure manometer to identify the level of collapse in obstructive sleep apnea. Otolaryngol Head Neck Surg 1992; 107: 651-655.

15. Katsantonis GP, Moss K, Miyazaki S, Walsh J. Determining the site of airway collapse in obstructive sleep apnea with airway pressure monitoring. Laryngoscope 1993; 103: 1126-1131.

16. Issa FG, Sullivan CE. Upper airway closing pressures in obstructive sleep apnea. J Appl Physiol 1984; 57: 520-527.

17. Rechtschaffen A, Kales A. A manual of standardized terminology, techniques and scoring system for sleep stages of human subjects. Washington DC, US government Printing Office, 1968.

18. Diagnostic Classification Steering Committee, Thorphy MT, Chairman. International Classification of Sleep Disorders: Diagnostic and Coding Manual. Rochester, Minnesota, American Sleep Disorders Association, 1990. 
19. Hudgel DW. The role of upper airway anatomy and physiology in obstructive sleep apnea. Clin Chest Med 1992; 13: 383-398.

20. McNicholas WT, Coffey M, McDonnel T, O'Regan R, Fitzgerald MX. Upper airway obstruction during sleep in normal subjects after selective oropharyngeal anesthesia. Am Rev Respir Dis 1987; 135: 1316-1319.

21. Berry KB, Kouchi KG, Bower JL, Light RW. Effect of upper airway anesthesia on obstructive sleep apnea. Am J Respir Crit Care Med 1995; 151: 1857-1861.

22. Pépin JL, Ferretti G, Lévy P. Voies aériennes supérieures et syndrome d'apnées du sommeil. Rev Mal Respir 1995; 12; 441-445.

23. Conradt R, Brandenburg U, Cassel W, Juhasz J, Peter $\mathrm{JH}$. Oesophageal pressure measurements - interference with sleep structure in nonapnoeic snorers. Eur Respir $J$ 1995; 8: 255s.

24. Skatvedt O, Akre H, Godtlibsen OB. Nocturnal polysomnography with and without continuous pharyngeal and esophageal pressure measurements. Sleep 1996; 19: 485489.

25. Sher AK, Schechtman KB, Piccirillo JF. The efficacy of surgical modifications of the upper airway in adults with obstructive sleep apnea syndrome. Sleep 1996; 19: 156-177.

26. Sforza E, Krieger J, Bacon W, Petiau C, Zamagni M, Boudewyns A. Determinants of effective continuous positive airway pressure in obstructive sleep apnea. Role of respiratory effort. Am J Respir Crit Care Med 1995; 151: $1852-1856$. 